\title{
Fabricación de fibras poliméricas a base de PLA obtenidas mediante electrohilado
}

\author{
Fabrication of PLA-based polymeric fibers obtained by \\ electrospinning
}

B. I. Persona-Báez ${ }^{1}$, C. A. Martínez-Pérez ${ }^{1}$, E. S. Zuñiga-Aguilar ${ }^{1}$

${ }^{1}$ Universidad Autónoma de Ciudad Juárez

\section{RESUMEN}

El electrohilado es una técnica que permite la obtención de fibras poliméricas en escala micro y nanométrica, las cuales son ampliamente utilizadas en ingeniería tisular y otras áreas biomédicas como biosensores y sistemas de liberación de fármacos, por mencionar algunos ejemplos. En la presenta nota técnica se presenta el método para la fabricación de un andamio a base de ácido poliláctico (PLA) y su caracterización por medio de Microscopía Electrónica de Barrido (SEM) y Espectroscopía de Transmisión de Infrarrojo con Transformada de Fourier (FTIR). Al aplicar dicho método de fabricación, se obtuvo un andamio cuyas fibras que oscilan de 7.5-200 nm presentan características favorables para su uso o aplicación en ingeniería tisular o medicina regenerativa. La alineación de las fibras puede significar una limitación para esta aplicación, sin embargo, ya se han reportado protocolos para mejorar esta característica en los andamios, por lo que la técnica de electrohilado es una de las mejores opciones en la fabricación de andamios porosos.

PALABRAS CLAVE: Electrohilado; PLA; ingeniería tisular; fibras poliméricas.

\section{ABSTRACT}

Electro-spinning is a technique that allows the obtaining of polymer fibers in micro and nano metric scales which are widely used in tissue engineering and other biomedical areas such as biosensors and drug delivery systems, to name a few examples. The present technical note presents the method for manufacturing a scaffold based on Poly-lactic acid (PLA) and its characterization by means of Scanning Electron Microscopy (SEM) and Fourier Transformed Infrared Transmission Spectroscopy (FTIR). When applying said manufacturing method, a scaffold was obtained whose fibers ranging from 7.5-200 nm have favorable characteristics for use or application in tissue engineering or regenerative medicine. Fiber alignment may mean a limitation for this application, however, protocols have already been reported to improve this feature on scaffolding. So the electro-spinning technique is one of the best options in the manufacture of porous scaffolding.

KEYWORDS: Electrospinning; PLA; tissue engineering; polymeric fibers.

Correspondencia:

DESTINATARIO: Esmeralda Sarai Zuñiga Aguilar INSTITUCIÓN: Universidad Autónoma de Ciudad Juárez DIRECCIÓN: Avenida del Charro 450 norte, col. Partido Romero, C. P. 32310, Ciudad Juárez, Chihuahua, México. CORREO ELECTRÓNICO: esmeralda.zuniga@uacj.mx
Fecha de recepción: 27 de enero de 2020. Fecha de aceptación: 5 de marzo de 2020 .

\begin{tabular}{|c|c|c|c|}
\hline (c) (1) \$ & & $\begin{array}{l}\text { Acceso } \\
\text { abierto }\end{array}$ & $\stackrel{\Delta}{\rightleftarrows}$ \\
\hline
\end{tabular}




\section{INTRODUCCIÓN}

En los últimos años, la ingeniería tisular se ha convertido en un tema de suma importancia puesto que se trata de una vía mediante la cual es posible restaurar, mantener o mejorar la función de órganos y tejidos ${ }^{[1]}$, aplicando conocimientos de una gran diversidad de disciplinas. Sin embargo, replicar la heterogeneidad espacial y funcional del tejido nativo sigue siendo un desafío ${ }^{[2]}$ que se puede enfrentar mediante el empleo de biomateriales, células, andamios y factores de crecimiento.

El término biomaterial es definido como un material destinado a interactuar con sistemas biológicos para tratar, aumentar o remplazar un tejido, órgano o función del cuerpo ${ }^{[3]}$. Estos se clasifican generalmente en metales, cerámicos, compuestos y polímeros. Cada uno de estos grupos poseen propiedades distintas, por lo que se les puede dar un adecuado uso dentro del ámbito biomédico. Los polímeros son los materiales más utilizados, de los cuales se fabrican implantes, materiales protésicos, sistemas de suministro de fármacos y productos de ingeniería tisular ${ }^{[4]}$, por mencionar algunos ejemplos.

Uno de los materiales poliméricos más utilizados es el ácido poliláctico (PLA, por sus siglas en inglés). Se trata de un poliéster alifático derivado del ácido láctico ${ }^{[5]}$. El PLA es biocompatible y su biodegradabilidad es posible por actividad enzimática e hidrólisis ${ }^{[6]}$. Además, al contar con excelentes propiedades mecánicas se convierte en una de las opciones más viables para múltiples aplicaciones biomédicas. Aunado a lo anterior, al ser un material moldeable, resulta sencillo otorgarle diferentes formas, entre las que se encuentran películas, nanopartículas, micelas y andamios ${ }^{[6]}$ utilizados en ingeniería de tejidos.

Los andamios son estructuras porosas que tienen la función de anclar las células y proporcionar un ambiente ideal para su crecimiento, proliferación y diferenciación ${ }^{[7]}$. Idealmente, deben presentar biocompatibilidad, biodegradabilidad, porosidad, interconectividad distribución de tamaño de poro adecuada y propiedades mecánicas apropiadas para su buen funcionamiento ${ }^{[8]}$. Debido a la importancia que han tomado en los últimos años, se han desarrollado técnicas mediante las cuales es posible fabricarlos.
Un buen ejemplo de dichas técnicas es el electrohilado, el cual destaca por su capacidad para otorgar ciertas ventajas como su versatilidad y rentabilidad ${ }^{[9]}$. Se trata de un método a través del cual es posible obtener fibras con diámetros que se encuentran en el orden de los micrómetros y nanómetros ${ }^{[10]}$. Los materiales fibrosos obtenidos presentan ventajas como un área superficial muy grande, altamente porosa, liviana y su funcionalidad puede ser alterada por funcionalización química y física ${ }^{[9]}$.

Durante el proceso, la aplicación de alto voltaje provoca que la fuerza del campo eléctrico supere las fuerzas de cohesión de la solución ${ }^{[11]}$. Los materiales poliméricos son utilizados porque son dieléctricos. Se pueden considerar como un arreglo de dipolos eléctricos microscópicos compuestos por cargas positivas y negativas cuyos centros no coinciden bajo la acción de un campo eléctrico ${ }^{[12]}$. Esto indica que cambian su posición como consecuencia de la aplicación de fuertes campos eléctricos externos ${ }^{[11]}$. De este modo, lo anterior da pie a una estructura denominada cono de Taylor, y así finalmente se logra la formación de la fibra. Esta emprende un viaje por el aire en el que permite la evaporación del disolvente y deja una fibra cargada que se desvía eléctricamente hasta su llegada a un colector metálico ${ }^{[13]}$.

Los parámetros que influyen en la morfología de las fibras obtenidas se pueden clasificar en parámetros de la solución, del proceso y ambientales. Estos son diversos y van desde el tipo de disolvente utilizado hasta el voltaje aplicado a la solución. Es importante destacar que la principal desventaja de este proceso es que no se encuentran estandarizados los parámetros que se deben utilizar para cada material. Así, al realizar un cambio en alguno de ellos es posible obtener andamios para distintos fines, ya sea en el campo de la ingeniería tisular o en otros como la liberación de fármacos, por mencionar algunos ejemplos.

En el presente trabajo se describe el proceso de obtención de fibras poliméricas mediante la técnica de electrohilado elaboradas a base PLA y su caracterización. Además, se establecen los parámetros ambientales, del proceso y de la solución necesarios para lograr la obtención de fibras de dicho material. 


\section{METODOLOGÍA}

Se preparó una solución polimérica a base de PLA Ingeo 3251D, obtenido de NatureWorks LLC, y cloroformo, de Fluka Analytical 31023, lote SZBD2340V, a una concentración de $13 \%(\mathrm{p} / \mathrm{v})$. Dicha solución se mantuvo en agitación magnética en un agitador Thermo Scientific ${ }^{\text {th }}$ Cimarec a temperatura ambiente por un lapso de $80 \mathrm{~min}$. Posteriormente a esta se le añadió 1 $\mathrm{mL}$ de etanol de Hycel, lote 428186, antes de comenzar el proceso de electrohilado. Luego, la solución fue cargada en una jeringa de $10 \mathrm{~mL}$ con una aguja de $0.6 \mathrm{~mm}$ de diámetro.

Por otro lado, en lo que respecta a la técnica de electrohilado, se utilizó un equipo de electrohilado de modelo único cuyo fabricante es Tamuse Systems-UACJ. Los parámetros utilizados fueron los siguientes: $20 \mathrm{kV}$ en la fuente de alimentación, una velocidad de flujo de 3 $\mathrm{mL} / \mathrm{h}$ y la velocidad del colector giratorio en $200 \mathrm{rpm}$. En cuanto a las condiciones ambientales, la temperatura ambiental se mantuvo y se trabajó con una humedad de $58 \%$. Las fibras se recolectaron en papel aluminio, el cual se puso en contacto físico con el colector cilíndrico del equipo de electrohilado conectado a la tierra de la fuente de alto voltaje.

Posteriormente, se realizó la caracterización física de las fibras obtenidas mediante Microscopía Electrónica de Barrido (SEM, por sus siglas en inglés). Esta se realizó con un equipo de SEM Hitachi modelo SU5000, con un voltaje de $20 \mathrm{kV}$, una distancia de trabajo analítica de $6.1 \mathrm{~mm}, 30 \mathrm{~Pa}$ de presión con 100 y 1000 magnificaciones. El proceso se llevó a cabo a temperatura ambiente.

Además, se realizó una caracterización con Espectroscopía de Transmisión de Infrarrojo con Transformada de Fourier (FTIR). El equipo utilizado fue el modelo NICOLET 6700, de Thermo Electron Corporation, el cual realiza un total de 32 mediciones a las cuales les calcula una media y se muestra al usuario.

\section{RESULTADOS Y DISCUSIÓN}

Se tomaron muestras de las fibras obtenidas con el objetivo de realizar una caracterización física utilizando SEM y FTIR. De la primera, se obtuvo la Figura 1, la cual fue procesada en el programa informático Image J para determinar el tamaño de las fibras y poros. En esta se observan fibras sin defectos, cuyos diámetros presen- tan tamaño promedio de $113 \mathrm{~nm}$, con una desviación estándar de $0.086 \mu \mathrm{m}$. El valor mínimo de diámetro es de $21 \mathrm{~nm}$ y el mayor es de $469 \mathrm{~nm}$. Los valores de los diámetros de las fibras se compararon con los obtenidos en un estudio de Campos y colaboradores ${ }^{[14]}$, en la que se utiliza una solución polimérica de PLA disuelto en cloroformo con parámetros similares a los empleados en el presente trabajo, como una concentración de $12.5 \%(\mathrm{p} / \mathrm{v}$ ) y un voltaje de $15 \mathrm{kV}$. Sin embargo, no se menciona el resto de los parámetros. Los resultados muestran semejanza, ya que se obtuvieron fibras desde $201 \mathrm{~nm}$ hasta $7.5 \mu \mathrm{m}$. El cambio en el diámetro puede ser adjudicado a diversos factores, como una distancia menor entre la aguja y el colector, menor conductividad de la solución y un mayor flujo de inyección ${ }^{[15]}$. Sin embargo, debido a la falta de información proporcionada en dicho estudio no es posible determinar la causa con exactitud.

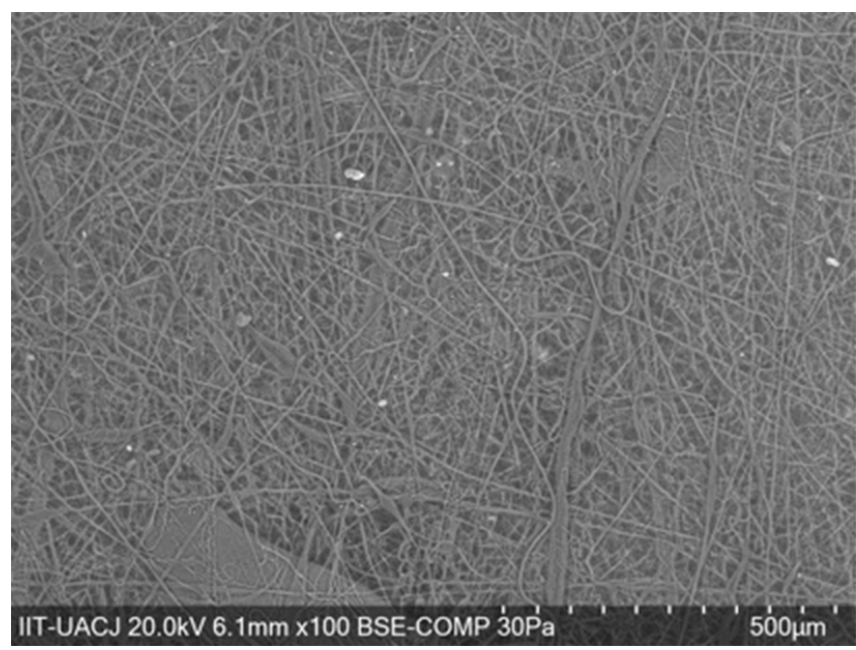

Figura 1. SEM de fibras de PLA con 100 magnificaciones.

En otro estudio realizado por León ${ }^{[16]}$ se obtuvo fibras de PLA disueltas en cloroformo por medio de electrohilado y se utilizaron los siguientes parámetros: voltaje de $20 \mathrm{kV}$ y velocidad de inyección de $0.5 \mathrm{~mL} / \mathrm{h}$. El resultado fue fibras con un diámetro promedio de 497 $\mathrm{nm}$, un mínimo de $215 \mathrm{~nm}$ y máximo de $1066 \mathrm{~nm}$. En comparación al presente estudio, el voltaje se mantuvo en $20 \mathrm{kV}$ en ambos casos. La velocidad de inyección o flujo fue distinto al empleado en este estudio, siendo menor el usado por León. Según la literatura ${ }^{[17,18]}$, esto que indica que a un menor flujo, el diámetro de las fibras es menor. Sin embargo, en este caso se observa lo contrario. De la misma forma que en la investigación elaborada por Campos y colaboradores, no es posible determinar si el diámetro de las fibras fue directamente 
afectado por la velocidad de inyección, ya que no se menciona el resto de los parámetros utilizados.

De la misma manera, se registró un tamaño de poro promedio de $25.05 \mu \mathrm{m}$ y una desviación estándar de $11.37 \mu \mathrm{m}$. El tamaño mínimo que se registró fue de 6 $\mu \mathrm{m}$ y el máximo de $63.31 \mu \mathrm{m}$. Los poros menores de $100 \mu \mathrm{m}$ benefician la adhesión celular, difusión de oxígeno y nutrientes y depuración de residuos ${ }^{[19]}$.

Con respecto al orden en el que se depositaron las fibras sobre el colector, no se presentó alineación, como se observa en la Figura 2. No obstante, ya existen investigaciones en las que se modifica la velocidad del colector para conseguir una mejor alineación ${ }^{[13,20,21]}$.

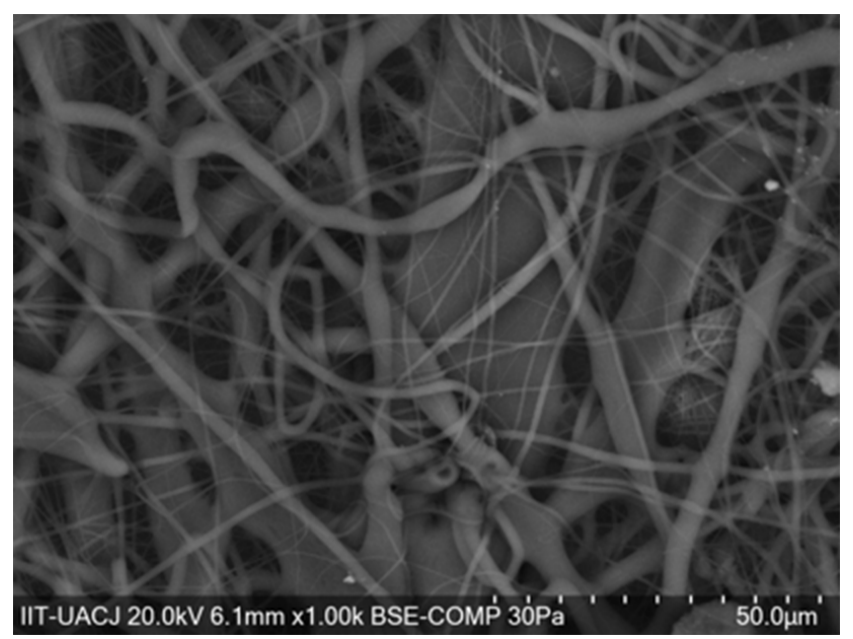

Figura 2. SEM de fibras electrohiladas de PLA con 1000 magnificaciones.
Los andamios fabricados para su uso en ingeniería tisular deben contar con las características necesarias para poder dirigirlos a un tejido en específico. Haciendo énfasis en el valor promedio de tamaño de poro del andamio de la Figura 1 y de acuerdo con una revisión literaria, se encontró que dicho valor es útil para células endoteliales y hepatocitos, por mencionar algunos ejemplos. Las primeras son romboidales y se disponen sobre la superficie interna de los vasos sanguíneos en donde actúa como barrera y elemento de unión entre la sangre y el resto de los tejidos ${ }^{[22]}$. Además, en promedio, las células endoteliales presentan un diámetro de $20 \mu \mathrm{m}^{[23]}$.

Por su parte, los hepatocitos presentan diferencias morfofuncionales dadas por su localización en el lóbulo hepático ${ }^{[24]}$. Sin embargo, se estima que el diámetro de estas células oscila entre $20-30 \mu{ }^{[25]}$. Resulta importante el tamaño de los últimos, ya que dentro de estos se llevan a cabo procesos como infiltración, migración, proliferación y transporte de fluidos ${ }^{[14]}$.

Por otro lado, en el gráfico de la Figura 3, obtenido mediante FTIR, se observan bandas características del PLA en $2929 \mathrm{~cm}^{-1}$, correspondiente al estiramiento de $\mathrm{C}-\mathrm{H}$, en $1753 \mathrm{~cm}^{-1}$ por el estiramiento de $\mathrm{C}=\mathrm{O}$ y en $1089 \mathrm{~cm}^{-1}$ debido al estiramiento de $\mathrm{C}-\mathrm{O}{ }^{[26]}$.

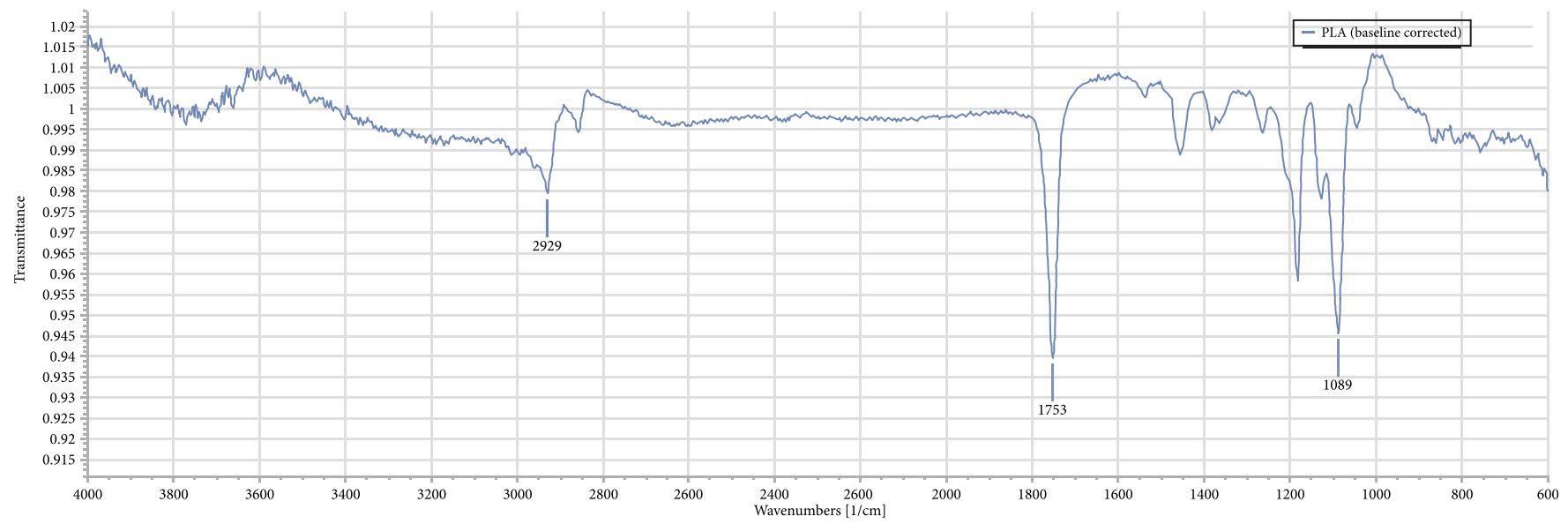

Figura 3. FTIR de fibras de PLA. 


\section{CONCLUSIONES}

La ingeniería tisular presenta avances a pasos agigantados, y esto se debe a los progresos tecnológicos que se han dado en las últimas décadas. El electrohilado se posiciona como una técnica sobresaliente en la fabricación de andamios, debido a la simplicidad del proceso en comparación a otros. Además, ofrece la posibilidad de obtener fibras con características deseadas mediante la modificación de parámetros que guardan una estrecha relación, con la finalidad de orientarla a distintos tejidos e incluso su asignación a otra aplicación biomédica.

\section{REFERENCIAS}

[1] L. G. Griffith y G. Naughton, "Tissue Engineering-Current Challenges and Expanding Opportunities", Science, vol. 295, no. 5557, pp. 1009-1014, 8 febrero 1998. doi: 10.1126/science.1069210

[2] D. K. Taheem, G. Jell y E. Gentleman, "Hypoxia Inducible Factor- $1 \alpha$ in Osteochondral Tissue Engineering", Tissue Engineering: Part B, vol. 0, no. 0, pp. 1-11, 9 enero 2020. doi: 10.1089/ten.teb.2019.0283

[3] A. Merolli y T. J. Joyce, eds. Biomaterials in hand surgery. Italia: Springer Science \& Business Media, 2009.

[4] P. Parida, A. Behera y S. C. Mishra, "Classification of Biomaterials used in Medicine", International Journal of Advances in Applied Sciences, vol. 1, no. 3, pp. 125-129, septiembre 2012.

[5] X. Pang, X. Zhuang, Z. Tang y X. Chen, "Polylactic acid (PLA): Research, development and industrialization", Biotechnology Journal, vol. 5, no. 11, pp. 1125-1136, 5 noviembre 2010. doi: 10.1002/biot.201000135

[6] B. Tyler, D. Gullotti, A. Mangraviti, T. Utsuki y H. Brem, "Polylactic acid (PLA) controlled delivery carriers for biomedical applications", Advanced Drug Delivery Reviews, vol. 107, pp. 163-175, 15 diciembre 2016. doi: 10.1016/j.addr.2016.06.018

[7] V. M. Pérez-Puyana, I. Carreño-Carmona, A. RomeroGarcía y A. F. Guerrero-Conejo, "Uso de biopolímeros para la elaboración de andamios con aplicación en ingeniería tisular", en IV Jornadas de Investigación $y$
Postgrado 2018 [en línea], Universidad de Sevilla, pp. 177-181. Disponible en: https://idus.us.es/bitstream/ handle/11441/88907/perez-puyana_ponencia_ sevilla_2018_uso.pdf? sequence $=1$ \&isAllowed $=y$

[8] C. Figueroa, R. Gómez, J. Prohíasy L. Pascual, “Obtención de andamios de colágeno para la restauración del tejido del miocardio", Revista Cubana de Ingeniería, vol. 7, no. 3, pp. 15-24, septiembre-diciembre 2016. Disponible en: http://rci.cujae.edu.cu/index.php/rci/article/ view/581/pdf

[9] A. Celebioglu y T. Uyar, "Electrospinning of cyclodextrins: hydroxypropyl-alpha-cyclodextrin nanofibers", Journal of Materials Science, vol. 55, no. 1, pp. 404-420, enero 2020. doi: 10.1007/s10853-019-03983-X

[10] S. Agarwal, J. H. Wendorff y A. Greiner, "Use of electrospinning technique for biomedical applications", Polymer, vol. 49, no. 26, pp. 5603-5621, 8 diciembre 2008. doi: 10.1016/j.polymer.2008.09.014

[11] L. M. Duque, L. Rodriguez y M. López, "Electrospinning: la era de las nanofibras", Revista Iberoamericana de Polímeros, vol. 14, no. 1, pp. 10-27, enero 2013. Disponible en: http://www.ehu.eus/reviberpol/pdf/ ENE13/duque.pdf

[12] A. J. Dekker, Electrical Engineering Materials. Englewood Cliffs, N. J.: Prentice-Hall, 1959.

[13] J. Kucinska-Lipka, I. Gubanska, H. Janik y M. Sienkiewicz, "Fabrication of polyurethane and polyurethane based composite fibres by the electrospinning technique for soft tissue engineering of cardiovascular system", Materials Science and Engineering: C, vol. 46, pp. 166-176, 1 enero 2015. doi: 10.1016/j.msec.2014.10.027

[14] J. Campos, L. Hurtado, N. Urdaneta, A. J. Cantanhede, R. N. Moreira Araújo y M. A. Sabino, "Micro-nanofibras de poli(ácido láctico) fabricadas por electrospinning y encapsulación de 2-[(E)-4-(dimetilamino) benzidileno)]indan-1-ona", Revista Latinoamericana de Metalurgia y Materiales, vol. 39, no. 2, pp. 94-104, 2019. Disponible en: https://www.rlmm.org/ojs/index.php/ rlmm/article/download/973/555

[15] Z.-M. Huang, Y.-Z. Zhang, M. Kotaki y S. Ramakrishna, "A review on polymer nanofibers by electrospinning 
and their applications in nanocomposites", Composites Science and Technology, vol. 63, no. 15, pp. 2223-2253, noviembre 2003. doi: 10.1016/S0266-3538(03)00178-7

[16] J. G. León, “Obtención de nanofibras Core/Shell de Ácido Poliláctico/Carboximetil Celulosa para su aplicación en liberación controlada de fármacos", tesis de maestría, Universidad Pedagógica y Tecnológica de Colombia, 2018. [En línea]. Disponible en: https://repositorio.uptc.edu.co/handle/001/2505

[17] X. Zong, K. Kim, D. Fang, S. Ran, B. S. Hsiao y B. Chu, "Structure and process relationship of electrospun bioabsorbable nanofiber membranes", Polymer, vol. 43, no. 16, pp. 4403-4412, julio 2002. doi: 10.1016/S00323861(02)00275-6

[18] C. Ribeiro, V. Sencadas, J. L. Gómez Ribelles y S. Lanceros-Méndez, "Influence of Processing Conditions on Polymorphism and Nanofiber Morphology of Electroactive Poly(vinylidene fluoride) Electrospun Membranes", Soft Materials, vol. 8, no. 3, pp. 274-287, 2010. doi: 10.1080/1539445X.2010.495630

[19] M. A. Sabino, M. Loaiza, J. Salazar, R. A. Rezende y J. V. Lopes Da Silva, "Obtención de andamios 3D biodegradables a doble escala usando técnicas de manufactura aditiva y electrospinning", Acta Macroscopica, vol. 24, supl. A, pp. 51-52, 2015. Disponible en: http:// actamicroscopica.ivic.gob.ve/Suplementos/Vol_24_Supp_A_2015_Memorias_CIASEM_2015/CIASEM 2015 CD/files/pdf/OS2-M-129-Sabino.pdf

[20] M. K. Leach, Z. Q. Feng, S. J. Tuck y J. M. Corey, “Electrospinning Fundamentals: Optimizing Solution and Apparatus Parameters", Journal of Visualized Experiments, no. 47, p. 2494, enero 2011. doi: 10.3791/2494

[21] M. Kim, Y. Kim, K. M. Lee, S. Y. Jung, E. Lee, S. H. Baeck y S. E. Shim, "Electrochemical improvement due to alignment of carbon nanofibers fabricated by electrospinning as an electrode for supercapacitor", Carbon, vol. 99, pp. 607-618, abril 2016. doi: 10.1016/j.carbon.2015.12.068

[22] J. Arias, M. Á. Aller, J. I. Arias y L. Lorente, Fisiopatología Quirúrgica. Traumatismos, Infecciones, Tumores, Editorial Tébar, 1999.

[23] M. Guerra Almaguer, J. A. Garza Chavarría, T. O. Tamargo Barbeito, T. Cárdenas Díaz, M. Río Torres, D. Cruz Izquierdo y Z. Pérez Parra, "Morfología y morfometría del endotelio corneal en adultos sin alteraciones corneales según cantidad de células evaluadas", Revista Cubana de Oftalmología, vol. 30, no. 3, julio-septiembre 2017. Disponible en: http://scielo.sld.cu/pdf/oft/v30n3/ oft04317.pdf

[24] J. Pacheco-Yépez, A. Rondán-Zárate, M. Shibayama, V. Tsutsumi y R. Campos-Rodríguez, "Daño a los hepatocitos por lectina inhibible por D-Galactosa/N-Acetil D-Galactosamina de Entamoeba Histolytica”, Revista del Centro de Investigación, vol. 7, no. 25, enero-junio 2006. Disponible en: http://revistasinvestigacion.lasalle.mx/index.php/recein/article/view/244/177

[25] V. Kegel, D. Deharde, E. Pfeiffer, K. Zeilinger, D. Seehofer y G. Damm, "Protocol for Isolation of Primary Human Hepatocytes and Corresponding Major Populations of Non-parenchymal Liver Cells", Journal of Visualized Experiments, no. 109, e53069, 30 marzo 2016. doi: $10.3791 / 53069$

[26] W. H. Hoidy, M. B. Ahmad, E. A. J. Al-Mulla y N. A. B. Ibrahim, "Preparation and characterization of polylactic acid/polycaprolactone clay nanocomposites", Journal of Applied Sciences, vol. 10, no. 2, pp. 97-106, 2010. doi: 10.3923/jas.2010.97.106 